\title{
18F-fluorodeoxyglucose (FDG) PET/CT after two cycles of neoadjuvant therapy may predict response in HER2-negative, but not in HER2- positive breast cancer
}

\author{
Jingyi Cheng ${ }^{1, *}$, Yujie Wang ${ }^{2,}{ }^{,}$, Miao $\mathrm{Mo}^{3}$, Xiao Bao ${ }^{4}$, Yingjian Zhang ${ }^{4}$, Guangyu Liu ${ }^{2}$, \\ Jun Zhang ${ }^{1}$, Daoying Geng ${ }^{1}$ \\ ${ }^{1}$ Department of Radiology, Huashan Hospital, Fudan University, Shanghai, P.R. China \\ ${ }^{2}$ Department of Breast Surgery, Key Laboratory of Breast Cancer in Shanghai, Fudan University Shanghai Cancer Center, \\ Department of Oncology, Shanghai Medical College, Fudan University, Shanghai, P.R. China \\ ${ }^{3}$ Clinical Statistics Center, Fudan University Shanghai Cancer Center, Department of Oncology, Shanghai Medical College, \\ Fudan University, Shanghai, P.R. China \\ ${ }^{4}$ Department of Nuclear Medicine, Fudan University Shanghai Cancer Center, Department of Oncology, Shanghai Medical \\ College, Fudan University Shanghai, P.R. China \\ *These authors have contributed equally to this work \\ Correspondence to: \\ Daoying Geng, e-mail: daoyinggeng@163.com \\ Jun Zhang, e-mail: zhj81828@163.com \\ Keywords: breast cancer, neoadjuvant therapy, trastuzumab, FDG PET/CT \\ Received: May 18, $2015 \quad$ Accepted: August 11, $2015 \quad$ Published: August 21, 2015
}

\section{ABSTRACT}

The aim of this prospective study was to assess the ability of $18 \mathrm{~F}$-fluorodeoxyglucose ( ${ }^{18} \mathrm{FDG}$ ) positron emission tomography/computed tomography (PET/CT) scanning to predict pathological complete response (PCR) in breast cancer, and to investigate whether timing of the scan and trastuzumab treatment influence the accuracy of PCR prediction in human epidermal growth factor receptor 2 (HER2) positive breast cancer patients. We treated 81 locally advanced breast cancer patients with four cycles of neoadjuvant chemotherapy (NAC). HER2-negative breast cancer patients received NAC alone, while HER2-positive breast cancer patients received NAC plus trastuzumab. ${ }^{18}$ FDG PET/CT scans were scheduled at baseline and after the second cycle of NAC. Axillary lymph node (ALN) dissection was performed after the last cycle of neoadjuvant therapy. Relative changes in standardized uptake values (SUV) between the two PET/ CT scans ( $\triangle$ SUV) in primary tumors and ALN metastases were calculated. There were 75 patients with $150 \mathrm{PET} / \mathrm{CT}$ scans in the final analysis, including 41 HER2-negative and 34 HER2-positive cases. In the HER2-negative group, the $\Delta$ SUV predicted overall and ALN PCR; the receiver operating characteristics-areas under curve (ROC-AUC) were 0.87 and 0.80 ( $P=0.0014$ and 0.031 , respectively $)$ and the negative predictive values were $94 \%$ and $89 \%$ respectively. However, in the HER2-positive group, $\triangle$ SUV could predict neither overall nor ALN PCR; the ROC-AUCs were only 0.56 and 0.53 , with $P=$ 0.53 and 0.84 , respectively. Hence, the $\Delta S U V$ after two cycles of neoadjuvant therapy could predict pCR in HER2-negative patients treated with NAC alone, but not in HER2positive patients treated with NAC plus trastuzumab.

\section{INTRODUCTION}

Preoperative neoadjuvant therapy is used as a standard treatment for locally advanced breast cancer to downstage the primary tumor and increase the breast conservation rate. Early prediction of success of neoadjuvant therapy is critical for determining whether a current treatment should be continued, stopped, or changed to a more aggressive regimen [1-3]. In recent years, several studies demonstrated that 18F-fluorodeoxyglucose ( $\left.{ }^{18} \mathrm{FDG}\right)$ positron emission tomography/computed tomography (PET/CT) is a useful 
tool for evaluating NAC response [4-6]. Relative change in the standardized uptake value $(\triangle \mathrm{SUV})$ after one or two cycles of NAC was reported to be a strong indicator of pathological response [7]. However, treatment options and responses to therapy differ greatly among various phenotypes. These differences complicate the utilization of $\triangle \mathrm{SUV}$ as a predictor of pathological responses [8-12].

This prospective study investigated the predictive reliability of ${ }^{18} \mathrm{FDG}$ uptake change in patients with human epidermal growth factor receptor 2 (HER2)-negative and HER2-positive phenotypes, particularly with respect to axillary lymph node (ALN) metastasis.

\section{RESULTS}

\section{Patients and pathologic response}

Eighty-one breast cancer patients were recruited: 3 were excluded because they missed follow-up (1 triplenegative breast cancer (TNBC) and 2 HER2-positive) and 3 HER2-positive patients underwent NAC only (including 1 luminal with HER[3+]) and 2 HER2-positive). Ultimately, 75 patients were included in the final analysis. Breast cancer was confirmed through core needle biopsy. Patients' tumor characteristics and treatments are listed in Table 1.

A majority of tumors were luminal $(52.0 \% ; 39 / 75)$, followed by HER2-positive $(30.6 \% ; 23 / 75)$, and TNBC $(17.3 \% ; 13 / 75)$. pCR occurred more often in patients with HER2 positive tumors $(52.1 \%)$ than in those with TNBC and luminal tumors (30.7\% and $28.2 \%$, respectively). Furthermore, the HER2-positive group treated with NAC plus trastuzumab showed higher pCR rates than the HER2-negative group treated with NAC alone (55.8\% and $19.5 \%$, respectively).

\section{$\triangle$ SUVpeak in primary lesions and metastatic lymph nodes}

A total of $150 \mathrm{PET} / \mathrm{CT}$ scans from 75 patients were eligible for analysis. Among them, 53 had ALN metastasis confirmed by both core needle biopsy and ${ }^{18} \mathrm{FDG}$ PET/CT. In the baseline PET/CT scan, the SUV peak of the primary lesions ranged from 22.2 to 1.60 (mean $=8.14 \pm 4.54$ ). The SUVpeak of ALN metastasis ranged from 15.87 to 1.20 (mean $=6.35 \pm 4.02$ ). On follow-up PET/CT scans after two cycles of neoadjuvant therapy, the SUVpeak of the primary lesions ranged from 9.60 to $0($ mean $=2.88 \pm 2.92)$, and that of ALN metastases ranged from 7.79 to $0($ mean $=1.67 \pm 1.76)$.

In the HER2-positive group, the SUVpeak of primary lesions fell from a baseline of $7.95 \pm 4.65$ to $2.06 \pm 3.01$ in the subsequent scan, a decrease of $73 \% \pm 32 \%$. Moreover, the SUVpeak of ALN metastasis fell from a baseline of 6.46 \pm 3.75 to $1.19 \pm 1.70$, a decrease of $84 \% \pm 18 \%$.

In the HER2-negative group, the SUVpeak of the primary lesions fell from a baseline of $8.28 \pm 4.49$ to 3.55 \pm 2.63 , a decrease of $52 \% \pm 33 \%$. The SUVpeak of ALN metastasis fell from $6.23 \pm 4.30$ to $2.20 \pm 1.70$, a decrease of $60 \% \pm 31 \%$. The $P$ values were 0.005 and 0.001 , respectively.

\section{$\triangle$ SUVpeak of primary lesions predict overall pCR}

In all 75 patients, ${ }^{18} \mathrm{FDG}$ PET/CT was performed before and after two cycles of neoadjuvant therapy. The SUVpeak obtained at these times could not predict overall pCR for primary lesions or ALN metastases. The $\triangle \mathrm{SUVpeak}$ of the primary lesion had a moderate predictive value with a receiver operator characteristics-area under the curve (ROC-AUC) of 0.75 (95\% confidence interval [CI] $0.65-0.86, P=0.001)$; the sensitivity and specificity were $78 \%$ and $69 \%$, respectively.

Of all cases, 34 were in the HER2-positive group that was treated with NAC and trastuzumab. ROC analysis showed that the $\triangle$ SUVpeak could not effectively predict the overall pCR because the ROC-AUC was only $0.56(95 \%$ CI $0.36-0.76, P=0.53)$. However, for the 41 patients of the HER2-negative group treated with NAC alone, ROC analysis showed that the $\triangle$ SUVpeak accurately predicted the overall pCR because the ROCAUC was 0.87 (95\% CI $0.75-0.98, P=0.0014)$. The sensitivity, specificity, positive predictive value, negative predictive value, and accuracy were $75 \%, 85 \%, 60 \%$, $94 \%$, and $83 \%$, respectively (Figure 1A, 1B).

In the HER2-negative group, we predicted $75 \%$ of pCRs $(6 / 8)$ and $88 \%$ of non-pCRs (29/33) by applying a cutoff value of $\triangle \mathrm{SUVpeak}>80 \%$. However, in the HER2positive group, the $\triangle \mathrm{SUV}$ peak could not predict pCR accurately because a clear cutoff could not be defined (Figure 2A, 2B).

\section{$\triangle$ SUV peak of ALN metastasis predicts pCR}

There were 53 patients with ALN metastasis confirmed by both core needle biopsy and ${ }^{18}$ FDG PET/ CT. Neither the baseline nor post two-cycle SUVpeak alone could predict ALN pCR. The $\triangle$ SUVpeak of the ALN metastases had a moderate predictive value with an ROC-AUC of 0.74 (95\% CI 0.60-0.88, $P=0.0021$ ). The sensitivity and specificity were $87 \%$ and $61 \%$, respectively.

Of all 75 cases, 28 were HER2-positive and were treated with NAC and trastuzumab. ROC analysis showed that the $\triangle$ SUVpeak of ALN metastasis could not predict the ALN pCR effectively because the ROC-AUC was only $0.53(95 \%$ CI $0.20-0.86, P=0.84)$. However, in the HER2-negative group of 25 cases treated with NAC alone, the $\triangle$ SUVpeak accurately predicted the ALN pCR, as the ROC-AUC was 0.80 (95\% CI 0.60-0.99, $P=0.031)$. The sensitivity, specificity, positive predictive value, negative predictive value, and accuracy were $67 \%, 89 \%, 67 \%$, $89 \%$, and $84 \%$, respectively (Figure 1C, 1D).

In the HER2-negative group, we could predict $66 \%$ of pCRs (4/6) and $89 \%$ of non-pCRs (17/19) by applying a cutoff value of $\triangle \mathrm{SUV}$ peak $>80 \%$. However, in the HER2positive group, the $\Delta$ SUVpeak could not accurately predict pCR because a clear cutoff value could not be defined (Figure 2C, 2D). 
Table 1: Patient and Tumor Characteristics $(n=75)$

\begin{tabular}{|l|c|l|}
\hline \multicolumn{3}{|c|}{ Age (years) } \\
\hline Mean & 43.7 & \\
\hline SD & 12.35 & \\
\hline Range & $24-65$ & \\
\hline
\end{tabular}

Tumor histology

\begin{tabular}{|l|c|c|}
\hline Ductal & 72 & $96.0 \%$ \\
\hline Lobular & 3 & $4.0 \%$ \\
\hline
\end{tabular}

Clinical tumor classification

\begin{tabular}{|l|c|c|}
\hline T1 & 10 & $13.3 \%$ \\
\hline $\mathrm{T} 2$ & 43 & $57.3 \%$ \\
\hline $\mathrm{T} 3$ & 19 & $25.3 \%$ \\
\hline $\mathrm{T} 4$ & 3 & $4.0 \%$ \\
\hline
\end{tabular}

Clinical lymph node classification

\begin{tabular}{|l|l|l|}
\hline N0 & 22 & $29.3 \%$ \\
\hline N1 & 39 & $52.0 \%$ \\
\hline N2 & 14 & $18.6 \%$ \\
\hline
\end{tabular}

primary lesions $(n=75)$

\begin{tabular}{|l|c|c|}
\hline $\mathrm{pCR}$ & 33 & $44.0 \%$ \\
\hline non-pCR & 42 & $56.0 \%$ \\
\hline
\end{tabular}

axillary lymph node metastasis $(n=53)$

\begin{tabular}{|l|c|c|}
\hline pCR & 30 & $56.6 \%$ \\
\hline non-pCR & 23 & $43.4 \%$ \\
\hline
\end{tabular}

Overall (primary + axillary lymph node metastasis) $(n=75)$

\begin{tabular}{|l|c|c|}
\hline pCR & 27 & $36.0 \%$ \\
\hline non-pCR & 48 & $64.0 \%$ \\
\hline HER2-positive group $(n=34)$ & pCR & non-pCR \\
\hline ER/PR $(-)$ HER2 $(+)(n=23)$ & 12 & 11 \\
\hline ER/PR $(+)$ HER2 $(+)(n=11)$ & 7 & non-pCR \\
\hline HER2-negative group $(n=41)$ & pCR & 9 \\
\hline ER/PR $(-)$ HER2 $(-)(n=13)$ & 4 & 24 \\
\hline ER/PR $(+)$ HER2 $(-)(n=28)$ & 4 & . \\
\hline
\end{tabular}

Abbreviations: SD, standard deviation; ER, estrogen receptor; PR, progesterone receptor. HER2, human epidermal growth factor receptor 2; NAC neoadjuvant chemotherapy; pCR, pathologic complete response.

\section{DISCUSSION}

The use of ${ }^{18} \mathrm{FDG}$ PET/CT imaging as an early predictive tool for breast cancer pCR has recently been encouraged. However, there has been controversy regarding the optimal timing of ${ }^{18} \mathrm{FDG}$ PET/CT scanning. Most researchers advocate performing ${ }^{18} \mathrm{FDG}$ PET/CT scanning halfway through chemotherapy $[13,14]$, while others suggest it be performed after the first cycle of chemotherapy $[15,16]$.

HER2-positive breast cancers usually undergo notable SUV changes during therapy, compared to other phenotypes. A 60\% change in SUV is easily achievable after the first cycle of chemotherapy. This degree of change ought to be sufficient for $\mathrm{pCR}$ prediction; therefore, some researchers suggest conducting ${ }^{18} \mathrm{FDG}$ after the first cycle 

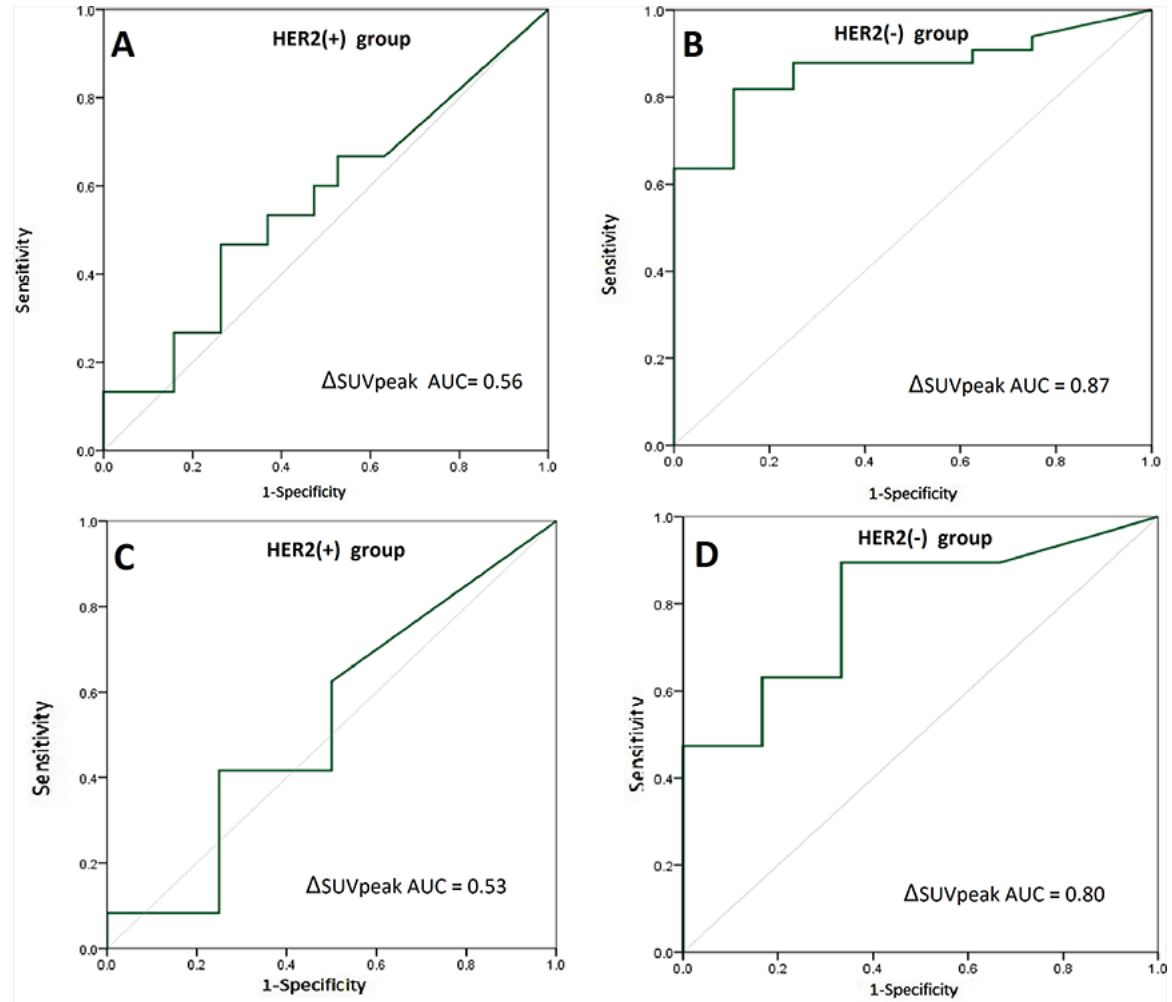

Figure 1: Receiver operating characteristics (ROC) analysis of $\triangle$ SUVpeak for the prediction of pathologic complete response (pCR). ROC analysis for prediction of overall pCR with the primary lesions' $\triangle$ SUVpeak in HER2-positive breast cancer A. and HER2-negative breast cancer B. ROC analysis for prediction of axillary lymph node (ALN) pCR with $\triangle$ SUVpeaks of ALN metastases in HER2-positive breast cancer $\mathbf{C}$. and HER2-negative breast cancer D. $\triangle$ SUVpeak refers to the difference in standard uptake values between baseline and after two chemotherapy cycles.

of chemotherapy to determine whether switching the therapeutic regimen is warranted [17].

We showed that $\triangle$ SUV after two cycles of neoadjuvan therapy accurately predicted the response in HER2-negative, but not HER2-positive patients. This appears to contradict existing studies. Actually, our result reveals the same view point on scanning timing for HER2positive from another aspect. The average degree of SUV change in the HER2-positive group was $73 \% \pm 32 \%$, which was much greater than the $52 \% \pm 33 \%$ observed in the HER2-negative group $(P=0.005$ for primary lesions and $P=0.001$ for ALN metastases). This suggests that the optimal ${ }^{18} \mathrm{FDG}$ PET-CT timing for the HER2-positive group, for purposes of predicting pCR, was missed.

Another reason is that the inflammation reaction caused by trastuzumab influences predictive accuracy. Firstly, in addition to directly inhibiting intracellular signaling in HER2-positive tumor cells, the combination with trastuzumab induces antibody-dependent cellular cytotoxicity (ADCC), which also contributes to changes in SUV [14]. Secondly, the decrease in ${ }^{18} \mathrm{FDG}$ uptake in patients receiving trastuzumab is not a pure reflection of cell killing but also reflects other specific effects on glucose metabolism, such as reductions in GLUT1 and hexokinase II activity $[18,19]$. As a result, breast lesion and ALN metastasis shrink and FDG uptake decrease greatly after two cycles of NAC due to higher sensitivity for NAC in HER2-positive group. At the same time, inflammation reaction caused by trastuzumab appears, causing the SUVs of residual lesions to increase and $\triangle \mathrm{SUV}$ s to therefore decrease. In cases with lower SUV baselines in particular, slight increases in SUV caused by inflammation could easily generate false negatives (Figure 3A-3D).

The response of breast cancer ALN metastases after NAC is of greater concern than that of the primary foci. In previous studies of HER2-positive cancers, the complete response rates of both primary foci and lymph nodes were quite high; hence, overtreatment can be avoided if ALN metastases show early pCR. Roussean et al. reported that the sensitivity and accuracy of therapeutic effect prediction for axillary lymph node metastases after chemotherapy were $96 \%$ and $84 \%$, respectively, with an SUV decrease of $50 \%$ set as the threshold [11]. Similarly, Koolen et al. reported that the specificity and positive predictive values were $95 \%$ and $86 \%$, respectively, if the SUV decrease was greater than $60 \%$ and the ROC-AUC was 0.80 [10]. There were also contradictory reports; Jung et al. reported that there was no correlation between SUV decrease and the pCR rate of lymph node metastasis [20]. In these reports, the breast cancer phenotypes were not considered, nor were the influences of therapies.

In our study, $\triangle \mathrm{SUV}$ was shown to be a more valuable predictor for HER2-negative patients who 

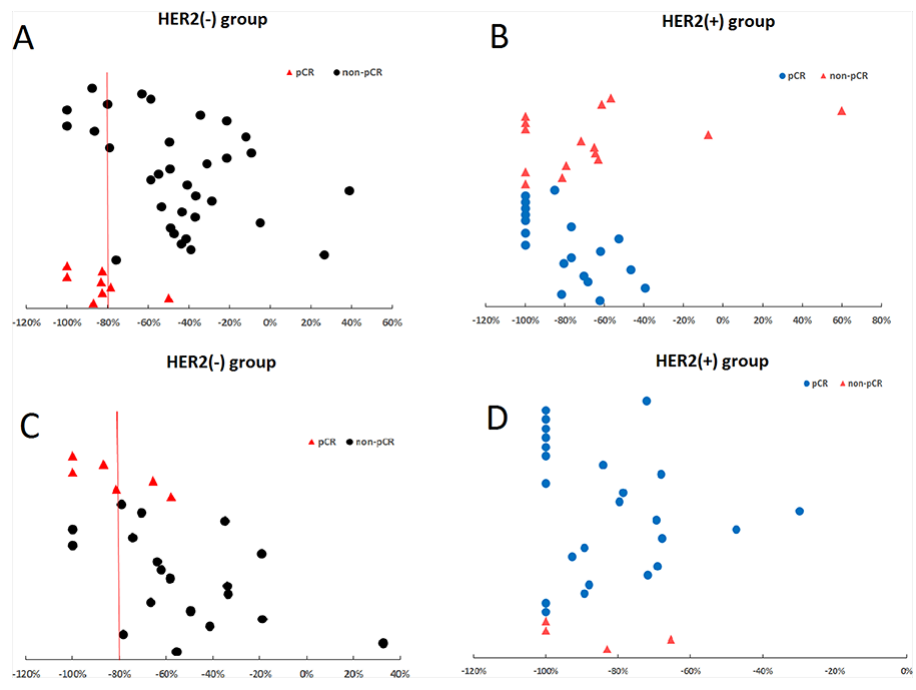

Figure 2: Pathological complete response (pCR) assessment in relation to the $\Delta$ SUVpeak. Application of a cutoff $\Delta$ SUVpeak of $>80 \%$ predicted $88 \%$ of overall non-pCRs A. and $89 \%$ of axillary lymph node (ALN) non-pCRs C. in the HER 2 -negative group, displaying higher negative predictive values of $94 \%$ and $89 \%$, respectively. In the HER2-positive group, $\triangle$ SUVpeak could not predict overall B. or ALN pCRs/non-pCRs D. because a clear cutoff could not be defined. $\triangle$ SUVpeak refers to the difference in standard uptake values between baseline and after two chemotherapy cycles.

underwent $\mathrm{NAC}$ alone ( $\mathrm{ROC}-\mathrm{AUC}=0.80, P=0.031$ ), while the ROC-AUC of the $\triangle \mathrm{SUV}$ decreased to $0.53(P=$ 0.84 ) in HER2-positive patients who received NAC plus trastuzumab. Determining a cutoff value of $\Delta$ SUVpeak $>$ $80 \%$ can predict $66 \%$ of pCR and $89 \%$ of non-pCR in HER2-negative patients. However, $\triangle \mathrm{SUV}$ peak did not accurately predict pCR in HER2-positive patients because a clear cutoff could not be established.

The main limitation of our study is the absence of SUV values after the first cycle of NAC; having multiple time points can better determine the best ${ }^{18} \mathrm{FDG}$ PET-CT timing for assessment.

In conclusion, this study shows that response monitoring of breast cancer patients with ${ }^{18}$ FDG PET-CT during neoadjuvan therapy is dependent on both phenotypes, therapeutic regimens and scan timing. For HER2- negative patients treated with NAC alone, the optimal timing is after tow cycles. $\triangle \mathrm{SUV}$ can accurately predict $\mathrm{pCR}$. Application of a cutoff $\triangle$ SUVpeak of $>80 \%$ predicted $88 \%$ of overall non-pCRs and $89 \%$ of ALN non-pCRs , displaying higher negative predictive values of $94 \%$ and $89 \%$, respectively. The clinical benefit of this study is that ${ }^{18}$ FDG PET-CT could offer the opportunity to switch to a different regimen for an individual patient who is likely to be non-pCR to NAC.

\section{MATERIALS AND METHODS}

\section{Patients}

To assess the potential value of ${ }^{18} \mathrm{FDG}$ PET/CT in predicting the response during neoadjuvant therapy, 81 breast cancer patients were recruited for this prospective study starting in February 2013. This study was approved by the Institutional Ethics Committee, and written informed consent was obtained from all patients. Enrolled were patients with newly diagnosed, non-inflammatory, and locally advanced (stage II and III) breast cancer who accepted neoadjuvant therapy. Clinical stage was determined according to the American Joint Committee on Cancer 6 th edition. Tumor size and T stage were assessed by clinical examination, ultrasonography, and/or magnetic resonance imaging (MRI). Diagnoses of invasive breast carcinoma and ALN metastasis were confirmed by core needle biopsy and fine needle aspiration, respectively, in all patients. Clinical stages were determined by mammography, ultrasonography, and MRI according to the TNM (tumor-node-metastasis) classification. Exclusion criteria were pregnancy, breast surgery, chemotherapy or radiotherapy history, diabetes, age younger than 18 years old, no ${ }^{18} \mathrm{FDG}$ uptake at baseline, or ineligibility for surgery.

\section{${ }^{18}$ FDG PET/CT Protocol}

${ }^{18} \mathrm{FDG}$ was produced by the Cyclotron (Siemens CTI RDS Eclipse ST) and Explora FDG4 modules at our center. All patients were required to fast for at least 6 hours to ensure glucose blood levels below $10 \mathrm{mmol} / \mathrm{L}$ (180 mg/dL), which was a prerequisite for undergoing the procedure. As long as the patient's blood glucose level was over $10 \mathrm{mmol} / \mathrm{L}(180 \mathrm{mg} / \mathrm{dL})$, the PET/CT exam should be cancelled. Before and after injection, patients lay comfortably in a quiet, dimly lit room. Scanning was initiated 1 hour after administration of the tracer $(7.4 \mathrm{MBq} / \mathrm{kg}$ or $0.2 \mathrm{mCi} / \mathrm{kg})$. The data acquisition procedure was as follows: CT scanning was first performed from the proximal thighs to head with $120 \mathrm{kV}$, CARE dose 4D mode, 80-250 mA, pitch 3.6. Intravenous or oral contrast was not used in CT scans. 

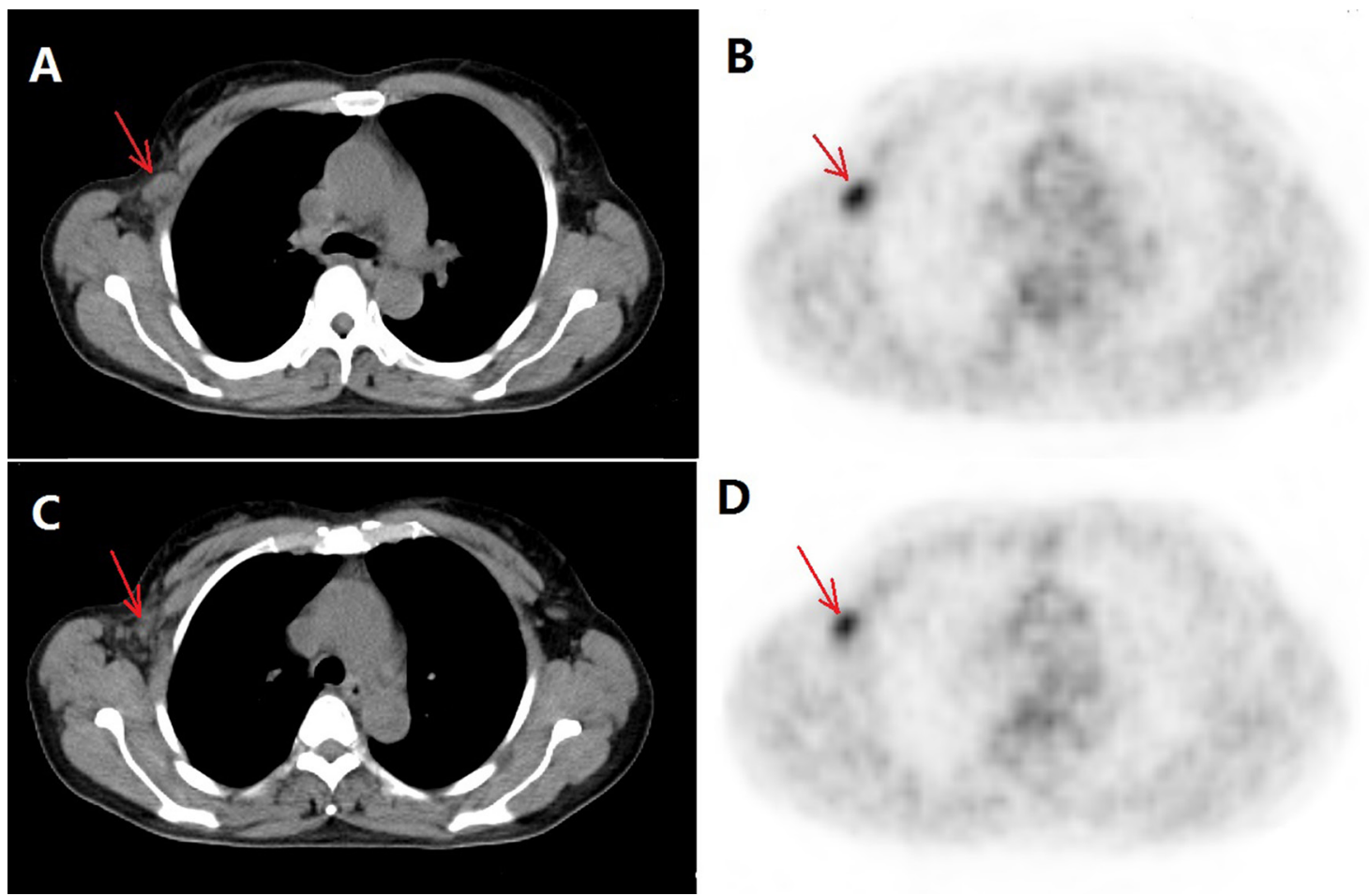

Figure 3: A false negative case involving a T2N1 breast primary lesion (HER2-positive) and right axillary lymph node metastasis before and after neoadjuvant chemotherapy (NAC) plus trastuzumab. Transverse slices of CT and PET images show that the size of the right axillary lymph node (ALN) metastasis is $2.3 \times 1.3 \mathrm{~cm}$ A. with high FDG uptake and a baseline SUVpeak of 5.16 B. After NAC plus trastuzumab treatment, the right ALN shrank C.; FDG uptake decreased and the SUVpeak was 2.72 D. The $\triangle$ SUVpeak is $-47 \%$ (from 5.16 to 2.72 ), less than the threshold of $-80 \%$, but the result of ALN dissection was pathological complete response. $\triangle$ SUVpeak refers to the difference in standard uptake values between baseline and after two NAC cycles.

Immediately after CT scanning, a PET emission scan that covered the identical transverse field of view was obtained. Acquisition time was $2-3$ min per table position. PET image data sets were reconstructed iteratively by applying the CT data for attenuation correction, and co-registered images were displayed on a workstation.

The baseline ${ }^{18}$ FDG PET/CT scans were scheduled prior to initiation of neoadjuvant therapy, at least ten days after core biopsy. The second scans were scheduled after the completion of the second cycle of neoadjuvant therapy just before commencing the third cycle. The same acquisition parameters were used for baseline and halfway studies. All the patients' PET examinations were done at the same center with the same equipment and methods. The PET/CT data was interpreted by two nuclear physicians blinded to clinical, radiologic, and pathologic findings.

\section{Image analysis}

Several lesions were evaluated, including primary lesions within the breast and regional lymph node metastases. All images were analyzed on a clinical Leonardo workstation with TrueD software. All the lesion volumes were defined by manually defining the volume of interest (VOI), referred to as the FDG-VOI on the ${ }^{18}$ FDG
PET/CT, and the hottest spot in the tumor foci was noted as the peak values of SUV (SUVpeak). After evidence of the initial ${ }^{18} \mathrm{FDG}$ hyper-metabolism subsided on the PET/ CT acquisition during neoadjuvant therapy, the VOI was copied onto the images of the second scans. The lesion with the highest initial uptake was assessed. The ALN to evaluate was defined as that having the most intense activity (SUVpeak $\geq 2.5$ ) in the axilla [21]. Relative changes in SUVpeak between two PET/CTs ( $\triangle \mathrm{SUVpeak})$ were calculated according to the following formula:

$\Delta$ SUVpeak $=[($ SUVpeak of second PET/CT SUVpeak of baseline PET/CT) / SUVpeak of baseline $\mathrm{PET} / \mathrm{CT}] \times 100 \%$.

\section{Histopathological analysis, definition of breast cancer subtypes and treatment}

Breast cancer diagnosis was performed via core-needle biopsy. Tumor grade was determined using the modified Scarff-Bloom-Richardson classification. Estrogen receptor (ER), progesterone receptor (PR), and HER2 status was determined on representative paraffin sections from each tumor using immunohistochemical (IHC) staining. ER and PR expression was considered positive if the number of positive nuclei was $>1 \%$. Cytoplasmic staining was not 
evaluated for consistency with the literature [22]. Positivity for the HER2 protein was evaluated according to the criteria of the HercepTest $[22,23]$. The HER2 membrane staining intensity and pattern were evaluated using the 0 to $3+$ scale. Scores of 0 and $1+$ (weak immunostaining in less than $10 \%$ of tumor cells) were defined as negative (HER2[-]), $2+$ (complete membrane staining in at least $10 \%$, but less than $30 \%$, of tumor cells) as equivocal, and $3+$ (uniformly intense membrane staining in at least $30 \%$ of tumor cells) as positive $($ HER2 $[+])$. Tumors with IHC scores of $3+$ and tumors with $2+$ that were further tested by fluorescence in situ hybridization (FISH) were reclassified as HER2 $(+)$. Tumors with IHC scores of 0 or $1+$ and tumors with IHC scores of $2+$ that were FISH-negative were classified as HER2(-).

Using IHC, the tumors were further classified into three subtypes:

- Luminal: ER and/or PR positive, HER2(-) or HER2(+) $(n=39)$.

- $\operatorname{HER} 2(+)$ : ER and PR negative, HER2 $(+),(n=23)$.

- TNBC: ER, PR, and HER2(-) $(n=13)$.

Different therapeutic regimens were administered according to subtypes and HER2 status:

- HER2(+) group: Luminal with HER2(3+) or HER2(2+) that were FISH-positive and HER2-positive $(n=34)$.

For HER2-positive patients, four weekly cycles of paclitaxel $80 \mathrm{mg} / \mathrm{m}^{2}$ and carboplatin at AUC of 2 in combination with trastuzumab $2 \mathrm{mg} / \mathrm{kg}$ (on days 1,8 , 15 , and 22; loading dose $4 \mathrm{mg} / \mathrm{kg}$ for week 1) were administered, and ALN dissection was performed less than 4 weeks after the last cycle of chemotherapy.

- HER2(-) group: Luminal with HER2(0), HER2(1+), or $\operatorname{HER}(2+)$ that were FISH negative and TNBC ( $n$ $=41$ ). Patients with HER2-negative breast cancer received four weekly cycles (12 doses over 16 weeks) of paclitaxel $80 \mathrm{mg} / \mathrm{m}^{2}$ and carboplatin at AUC of 2 (both on days 1, 8, and 15 of a 28-day cycle) followed by ALN dissection surgery.

\section{Pathology assessment}

pCR was defined as the absence of residual invasive cancer on hematoxylin and eosin evaluation of the complete resected breast specimen and all sampled regional lymph nodes following completion of neoadjuvant systemic therapy. Absence of in situ carcinoma was not required to define pCR [24].

\section{Statistical analysis}

Data was analyzed using the SPSS 22.0 software. Correlation between texture parameters was analyzed using Person's test. The predictive performance regarding the identification of responders or non-responders was evaluated by using univariate ROC analysis. The ROCAUCs were compared using the $Z$ test. $P<0.05$ was considered statistically significant.

\section{ACKNOWLEDGMENTS}

We thank all the subjects of this study for their participation. We greatly appreciate Wen-Tao Yang at Department of Pathology of Shanghai Cancer Center and Jian-Ping Zhang, Chun-lei Su, Zhi-feng Yao at Department of Nuclear Medicine for their excellent assistance.

\section{CONFLICTS OF INTEREST}

None declared.

\section{GRANT SUPPORT}

This research is supported by grants from the National Natural Science Foundation of China (NSFC81 301246,81201071,81471627) and the Outstanding Youth Grant from the Shanghai Municipal Commission of Health and Family Planning (XYQ2013107). This research is supported by the funders that had no role in study design, data collection and analysis, decision to publish, or preparation of the manuscript.

\section{REFERENCES}

1. Wolmark N, Wang J, Mamounas E, Bryant J, Fisher B. Preoperative chemotherapy in patients with operable breast cancer: Nine-year results from national surgical adjuvant breast and bowel project B18. J Natl Cancer Inst Monogr. 2001; 30:96-102.

2. Rastogi P, Anderson SJ, Bear HD, Geyer CE, Kahlenberg MS, Robidoux A, Margolese RG, Hoehn JL, Vogel VG, Dakhil SR, Tamkus D, King KM, Pajon ER, et al. Preoperative chemotherapy: Updates of national surgical adjuvant breast and bowel project protocols B-18 and B-27. J Clin Oncol. 2008; 26:778-785.

3. Mauri D, Pavlidis N, Ioannidis JP. Neoadjuvant versus adjuvant systemic treatment in breast cancer: A meta-analysis. J Natl Cancer Inst. 2005; 97:188-194.

4. Shelling BM, Avril N, Nahrig J, Kuhn W, Römer W, Sattler D, Werner M, Dose J, Jänicke F, Graeff H, Schwaiger M. Positron emission tomography using [18F] fluorodeoxyglucose for monitoring primary chemotherapy in breast cancer. J Clin Oncol. 2000; 18:1689-1695.

5. Rousseau C, Deviller A, Sagan C, Ferrer L, Bridji B, Campion L, Ricaud M, Bourbouloux E, Doutriaux I, Clouet M, Berton-Rigaud D, Bouriel C, Delecroix V, et al. Monitoring of early response to neoadjuvant chemotherapy in stage II and III breast cancer by $[18 \mathrm{~F}]$ fluorodeoxyglucose positron emission tomography. J Clin Oncol. 2006; 24:5366-5372.

6. Hatt M, Groheux D, Martineau A, Espié M, Hindié E, Giacchetti S, de Roquancourt A, Visvikis D, Cheze-Le Rest C. Comparison between 18F-FDG PET image-derived indices for early prediction of response to neoadjuvant chemotherapy in breast cancer. J Nucl Med. 2013; 54:341-349. 
7. Buchbender C, Kuemmel S, Hoffmann O, Stahl AR, Kimmig R, Otterbach F, Ladd S, Koeninger A, Forsting M, Bockisch A, Antoch G, Heusner TA. FDG-PET/CT for the early prediction of histopathological complete response to neoadjuvant chemotherapy in breast cancer patients: initial results. Acta Radiol. 2012; 53:628-636.

8. Koolen BB, Pengel KE, Wesseling J, Vogel WV, Vrancken Peeters MJ, Vincent AD, Gilhuijs KG, Rodenhuis S, Rutgers EJ, Valdés Olmos RA. FDG PET/CT during neoadjuvant chemotherapy may predict response in ER-positive/ HER2-negative and triple negative, but not in HER2positive breast cancer. Breast. 2013; 22:691-697.

9. Coudert B, Pierga JY, Mouret-Reynier MA, Kerrou K, Ferrero JM, Petit T, Kerbrat P, Dupré PF, Bachelot T, Gabelle P, Giard S, Coeffic D, Bougnoux P, et al. Use of $\left[{ }^{18} \mathrm{~F}\right]$-FDG PET to predict response to neoadjuvant trastuzumab and docetaxel in patients with HER2-positive breast cancer, and addition of bevacizumab to neoadjuvant trastuzumab and docetaxel in -FDG PET-predicted nonresponders (AVATAXHER): an open-label, randomized phase 2 trial. Lancet Oncol. 2014; 15:1493-1502.

10. Koolen BB, Valdés Olmos RA, Wesseling J, Vogel WV, Vincent AD, Gilhuijs KG, Rodenhuis S, Rutgers EJ, Vrancken Peeters MJ. Early assessment of axillary response with ${ }^{18} \mathrm{~F}$-FDG PET/CT during neoadjuvant chemotherapy in stage II-III breast cancer: implications for surgical management of the axilla. Ann Surg Oncol. 2013; 20:2227-2235.

11. Rousseau C, Devillers A, Campone M, Campion L, Ferrer L, Sagan C, Ricaud M, Bridji B, Kraeber-Bodéré F. FDG PET evaluation of early axillary lymph node response to neoadjuvant chemotherapy in stage II and III breast cancer patients. Eur J Nucl Med Mol Imaging. 2011; 38:1029-1036.

12. Machida Y, Kubota K, Katayama T, Toriihara A, Shibuya H. Diagnostic performance of fluorodeoxyglucose-positron emission tomography/computed tomography combined with ultrasonography-guided fine needle aspiration cytology for identifying axillary lymph node status in patients with breast cancer. Eur J Surg Oncol. 2013; 39:26-30.

13. Groheux D, Giacchetti S, Hatt M, Marty M, Vercellino L, de Roquancourt A, Cuvier C, Coussy F, Espié M, Hindié E. HER2-positivesing breast cancer: FDG uptake after two cycles of chemotherapy predicts the outcome of neoadjuvant treatment. Br J Cancer. 2013; 109:1157-1164.

14. Gebhart G, Gámez C, Holmes E, Robles J, Garcia C, Cortés M, de Azambuja E, Fauria K, Van Dooren V, Aktan G, CocciaPortugal MA, Kim SB, Vuylsteke P, et al. 18F-FDG PET/CT for early prediction of response to neoadjuvant lapatinib, trastuzumab, and their combination in HER2-positive breast cancer: results from Neo-ALTTO. J Nucl Med. 2013; 54:1862-1868.

15. Keam B, Im SA, Koh Y, Han SW, Oh DY, Cho N, Kim JH, Han W, Kang KW, Moon WK, Kim TY, Park IA, Noh DY, et al. Early metabolic response using FDG PET/CT and molecular phenotypes of breast cancer treated with neoadjuvant chemotherapy. BMC Cancer. 2011; 11:452-461.
16. Humbert $\mathrm{O}$, Berriolo-Riedinger A, Riedinger JM, Coudert B, Arnould L, Cochet A, Loustalot C, Fumoleau P, Brunotte F. Changes in $18 \mathrm{~F}-\mathrm{FDG}$ tumor metabolism after a first course of neoadjuvant chemotherapy in breast cancer: influence of tumor subtypes. Ann Oncol. 2012; 23:2572-2577.

17. Coudert BP, Largillier R, Arnould L, Chollet P, Campone M, Coeffic D, Priou F, Gligorov J, Martin X, Trillet-Lenoir V, Weber B, Bleuse JP, Vasseur B, et al. Multicenter phase II trial of neoadjuvant therapy with trastuzumab, docetaxel, and carboplatin for human epidermal growth factor receptor-2-positivesing stage II or III breast cancer: results of the GETN(A)-1 trial. J Clin Oncol. 2007; 25:2678-2684.

18. Smith TA, Appleyard MV, Sharp S, Fleming IN, Murray K, Thompson AM. Response to trastuzumab by HER2 expressing breast tumour xenografts is accompanied by decreased hexokinase II, glut1 and [(18)F]-FDG incorporation and changes in (31)P-NMR-detectable phosphomonoesters. Cancer Chemother Pharmacol. 2013; 71:473-480.

19. Shah C, Miller TW, Wyatt SK, McKinley ET, Olivares MG, Sanchez V, Nolting DD, Buck JR, Zhao P, Ansari MS, Baldwin RM, Gore JC, Schiff R, et al. Imaging biomarkers predict response to Anti-HER2 (ErbB2) therapy in preclinical models of breast cancer. Clin Cancer Res. 2009; 15:4712-4721.

20. Jung SY, Kim SK, Nam BH, Min SY, Lee SJ, Park C, Kwon Y, Kim EA, Ko KL, Park IH, Lee KS, Shin KH, Lee S, et al. Prognostic impact of [18F] FDG-PET in operable breast cancer treated with neoadjuvant chemotherapy. Ann Surg Oncol. 2010; 17:247-253.

21. Straver ME, Aukema TS, Olmos RA, Rutgers EJ, Gilhuijs KG, Schot ME, Vogel WV, Peeters MJ. Feasibility of FDG PET/ CT to monitor the response of axillary lymph node metastases to neoadjuvant chemotherapy in breast cancer patients. Eur J Nucl Med Mol Imaging. 2010; 37:1069-1076.

22. Liu Y, Huang X, Bi R, Yang W, Shao Z. Similar prognoses for invasive micropapillary breast carcinoma and pure invasive ductal carcinoma: a retrospectively matched cohort study in China. PLoS One. 2014; 9:e106564.

23. Wolff AC, Hammond MEH, Schwartz JN, Hagerty KL, Allred DC, Cote RJ, Dowsett M, Fitzgibbons PL, Hanna WM, Langer A, McShane LM, Paik S, Pegram MD, et al. American Society of Clinical Oncology/College of American Pathologists Guideline Recommendations for Human Epidermal Growth Factor Receptor 2 Testing in Breast Cancer. J Clin Oncol. 2007; 25:118-145.

24. US Food and Drug Administration . Pathologic complete response in neoadjuvant treatment of high-risk early-stage breast cancer: Use as an endpoint to support accelerated approval. 2014; URL: http://www.fda.gov/ downloads/drugs/guidancecomplianceregulatoryinforma tion/guidances/ucm305501.pdf [Accessed July 12 2015]. 